\title{
Métodos de cálculo para estimar la evapotranspiración de referencia para el Valle de Tumbaco
}

\section{Calculation methods of the reference evapotranspiration for the Tumbaco Valley}

\author{
Randon Ortiz C. ${ }^{{ }^{*}}$, Maritza Chile A. ${ }^{1}$ \\ ${ }^{1}$ Universidad Central del Ecuador. Facultad de Ciencias Agrícolas. Jerónimo Leiton y Av. La Gasca s/n. Ciudadela Universitaria. \\ 17052. Quito, Ecuador.
}

Autor para correspondencia: rsortiz@uce.edu.ec

\begin{abstract}
Resumen
La estimación precisa de la evapotranspiración de referencia permite obtener valores más reales sobre las necesidades hídricas de los cultivos. El objetivo del estudio consistió en evaluar nueve métodos a fin de estimar la evapotranspiración de referencia para las condiciones ambientales del Valle de Tumbaco, ubicado en la provincia de Pichincha (Ecuador). Se utilizó información climatológica mensual de la estación La Tola para determinar ET. Los métodos analizados fueron: FAO56, Tanque evaporímetro, Thornthwaite modificado, Hargreaves, Jensen-Haise, Makkink, Priestley-Taylor, Turc y FAO Radiación. Se calcularon los siguientes parámetros inferenciales para el análisis de los resultados, tomando como base FAO56: error medio absoluto, coeficiente de determinación, coeficiente de concordancia y coeficiente de confiabilidad. Los resultados principales fueron: i) el método FAO Radiación presentó una precisión buena; ii) los métodos Jensen and Haise, Makkink y Priestley and Taylor, presentaron índices tolerables; iii) los métodos del tanque evaporímetro, Thornthwaite modificado, Hargreaves y Turc proporcionaron índices inferenciales muy pobres. En conclusión, el método que debe utilizarse para determinar $\mathrm{ET}_{\mathrm{o}}$ mensual para el entorno agroecológico del Valle de Tumbaco es FAO56.
\end{abstract}

Palabras clave: Penman-Monteith, necesidades hídricas de los cultivos, balance hídrico, Ecuador

\begin{abstract}
The precise determination of the reference evapotranspiration ensures computing real values of the crop water requirements. The objective of the study was to evaluate nine methods to determine the reference evapotranspiration for the environmental conditions of the Tumbaco Valley located in the province of Pichincha (Ecuador). Monthly climatological data from La Tola station was used to estimate ET. The methods used to determine $\mathrm{ET}_{\mathrm{O}}$ were FAO56, Class A pan, modified Thornthwaite, Hargreaves, Jensen - Haise, Makkink, Priestley - Taylor, Turc and FAO Radiation. The following inferential parameters were used in the analysis, considering FAO56 as base: the absolute mean error, the coefficient of determination, the concordance coefficient and the confident coefficient. The results for monthly average climatological data showed that: i) the FAO Radiation method presented good statistical indexes; ii) the methods Jensen and Haise, Makkink and Priestley - Taylor presented poor indexes; iii) the methods Class A pan, modified Thornthwaite, Hargreaves and Turc presented the lowest inferential values. In conclusion, the method that should be used to estimate monthly $\mathrm{ET}_{\mathrm{o}}$ for the agro ecological environment of the Tumbaco Valley with good accuracy is FAO56.
\end{abstract}

Keywords: Penman-Monteith, irrigation requirements, water budget, Ecuador 


\section{Introducción}

La evapotranspiración de referencia $\left(\mathrm{ET}_{\mathrm{o}}\right)$ es un parámetro muy importante en el cálculo de los requerimientos de agua de los cultivos; este parámetro constituye la columna vertebral del diseño agronómico de todo sistema de riego, a través del cual se dimensionan redes de canales, redes de tuberías, reservorios; además, facilita la planificación de la operación de un sistema de riego (calendario y turnos de riego), y permite planificar la gestión de los recursos hídricos en una cuenca. Una sobreestimación de $\mathrm{ET}_{\mathrm{o}}$ producirá diseños de obras sobredimensionadas con costos elevados (construcción y mantenimiento), y una subestimación, en cambio, diseños de obras muy pequeñas, las cuales no permitirán cubrir la demanda de agua de los cultivos. Con relación al manejo del agua de riego, una sobreestimación de $\mathrm{ET}_{\mathrm{o}}$ en 1,0 mm, influirá en el incremento del volumen utilizado de agua en $10 \mathrm{~m}^{3}$ $\mathrm{ha}^{-1}$ en cada riego, encareciendo los costos de producción y generando condiciones favorables para el desarrollo de enfermedades fungosas, de tal manera que, para optimizar el uso del agua de riego se requiere estimar $\mathrm{ET}_{\mathrm{o}}$ con precisión.

A pesar de la variabilidad temporal y espacial del clima, se han implementado diferentes metodologías para determinar la $\mathrm{ET}_{\mathrm{o}}$, las cuales se han desarrollado empíricamente a través de experimentos de campo y con base en aproximaciones teóricas (Landon, 2004), entre éstas se pueden mencionar: el tanque evaporímetro y fórmulas empíricas; dentro de las fórmulas se tiene: modelo físico completo (Penman-Monteith); basados en la temperatura (Thornthwaite y Turc); basados en la temperatura y en la radiación solar (Hargreaves, Jensen and Haise, Makkink, Priestley and Taylor y FAO Radiación), entre otros métodos (Allen et al., 1990; Bhabagrahi et al., 2012). El método más completo para estimar $\mathrm{ET}_{\mathrm{o}}$ es Penman-Monteith FAO56 (Allen et al., 2006), el cual únicamente puede utilizarse en sitios en donde existe la suficiente información climatológica que demanda este método, en tanto que los otros métodos pueden utilizarse en zonas con muy poca información climatológica, previo a calibraciones locales.

La Organización de las Naciones Unidas para la Agricultura y la Alimentación (FAO) recomienda utilizar el método estándar Penman-Monteith, descrito en la publicación No. 56, para determinar
$\mathrm{ET}_{\mathrm{o}}$, debido a que este método se ajusta tanto a zonas áridas como templadas y tropicales (Allen et al., 2006). Este método determina $\mathrm{ET}_{\mathrm{o}}$ con base en tres componentes: i) resistencia aerodinámica (demanda evaporativa de la atmósfera estimada en función de las variables climatológicas: temperatura promedio, máxima y mínima, humedad relativa, horas de luz solar, viento, altitud y latitud); ii) resistencia superficial del cultivo (flujo del agua por difusión desde las raíces hasta los estomas de la planta y de la evaporación directa del agua desde el suelo), y iii) albedo (radiación solar reflejada por el cultivo); para el cultivo de referencia (pasto) de una altura de $0,12 \mathrm{~m}$, bien regado y con una cobertura total de la superficie del suelo, la resistencia superficial estimada es de $70 \mathrm{~s} \mathrm{~m}^{-1}$ y la radiación solar reflejada o albedo de 0,23 (Allen et al., 2006).

El tanque evaporímetro Clase A proporciona una medida integrada del efecto de la radiación, viento, temperatura y humedad sobre el proceso evaporativo que ocurre en una superficie libre de agua, a pesar de que existen varios factores que difieren entre la evaporación y la transpiración, como: reflexión de la radiación solar, almacenamiento de calor en el agua contenida en el tanque, turbulencia alrededor del tanque, transferencia de calor a través de las paredes del tanque y localización (variables que influyen en el balance energético); el tanque evaporímetro puede utilizarse para estimar la $\mathrm{ET}_{\text {o }}$ en forma confiable cuando las lecturas de la lámina de agua se realizan correctamente. La ET se determina a través de la utilización de un coeficiente empírico derivado de las condiciones físicas y ambientales que rodean al tanque, el cual varía entre 0.40 y 0.85 (Allen et al., 2006; Doorenbos \& Pruitt, 1977). El método de Thornthwaite fue desarrollado para el territorio de los Estados Unidos con base en el índice de calor anual a partir de la temperatura promedio mensual (Thornthwaite, 1948), este método fue modificado por Camargo et al. (1999). El método Hargreaves fue diseñado para condiciones ambientales de California (Hargreaves \& Samani, 1985). El método Makkink es una modificación de la ecuación de Penman, después de haber comparado los resultados proporcionados por el método Penman con valores obtenidos a través de lisímetros para condiciones ambientales de Holanda (Allen et al., 1990; Landon, 2004). El método Priestley and Taylor es una versión reducida de la ecuación combinada original de Penman y adaptada para zonas 
húmedas como el estado de Victoria en Australia (Priestley \& Taylor, 1972). La fórmula de Turc fue desarrollada para el clima del oeste de Europa (Landon, 2004). El método FAO Radiación es una modificación de la fórmula de Makkink, sugerido para utilizarse en zonas donde al menos exista la información de la temperatura del aire (Doorenbos \& Pruitt, 1977).

En Ecuador, muchos estudios de balance hídrico se han realizado con $\mathrm{ET}_{\mathrm{o}}$ calculada a través del método de Thornthwaite modificado, tal es así que, el Plan Nacional de la gestión integrada de los recursos hídricos de las cuencas y microcuencas hidrográficas del Ecuador se realizó utilizando la ET oalculada con este método, considerando que el Instituto Nacional de Meteorología e Hidrología determinó que este método y Penman-Monteith presentaron la misma tendencia para las condiciones ambientales del Ecuador (CISPDR, 2016).

A pesar de que en el Centro Académico Docente Experimental La Tola (CADET) se producen cultivos con fines comerciales y se desarrollan proyectos de investigación de diverso tipo, no se cuenta con una metodología adaptada a la zona que permita determinar $\mathrm{ET}_{\mathrm{o}}$ con precisión para calcular las necesidades hídricas y programar la aplicación del agua de riego a los cultivos, cualquiera que sea su método de aplicación.

El objetivo del estudio consistió en evaluar nueve métodos para determinar la evapotranspiración de referencia en el Valle de Tumbaco en Ecuador, con el fin de optimizar el uso del agua de riego.

\section{Materiales y métodos}

\section{1 Área de estudio}

El área de estudio para la presente investigación se estableció en el Valle de Tumbaco, localizado en la República del Ecuador, provincia de Pichincha, ubicado en la cuenca alta del río Esmeraldas. Se utilizó información de la estación agrometeorológica La Tola (M002) perteneciente al Instituto Nacional de Meteorología e Hidrología (INAMHI), localizada a una altura de $2480 \mathrm{~m}$, a $0^{\circ} 13^{\prime} 46^{\prime \prime}$ de latitud sur y $78^{\circ} 22^{\prime} 0^{\prime \prime}$ "de longitud oeste (fFgura 1). La serie histórica mensual de datos va desde 1986 hasta 2018 (32 años).

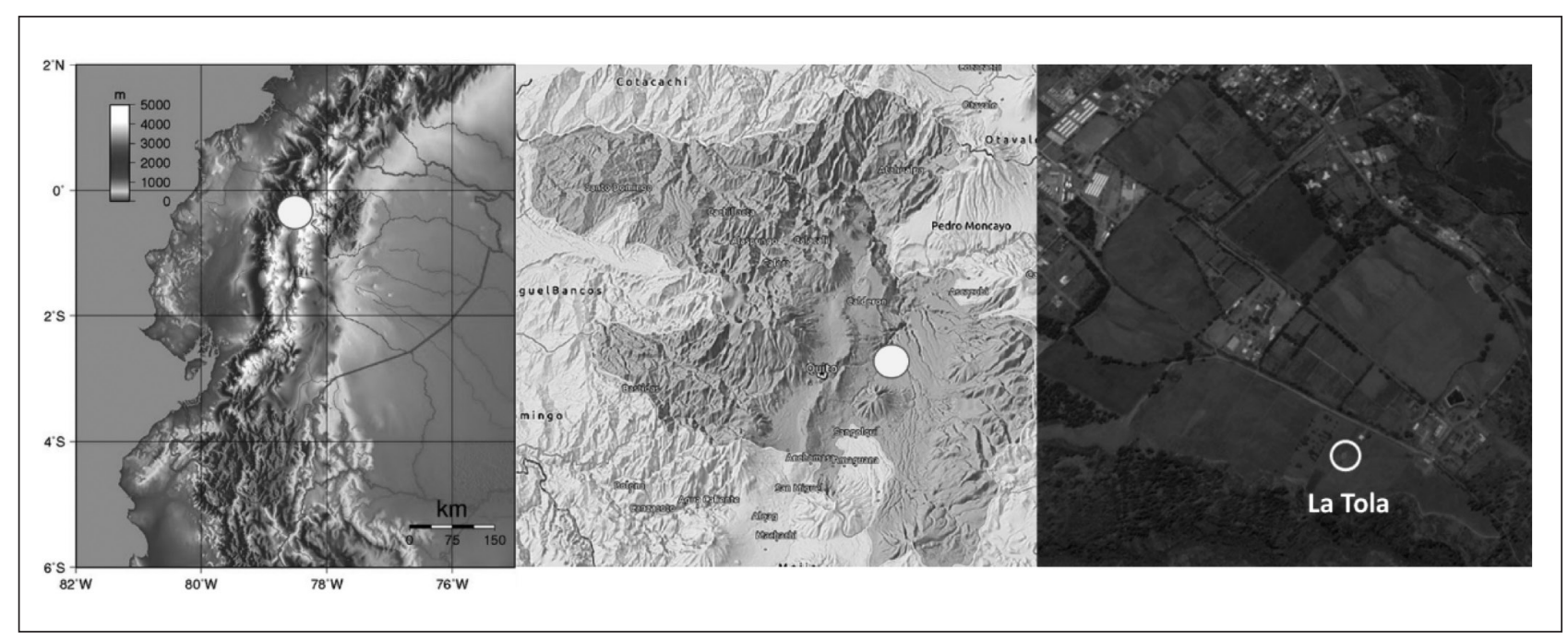

Figura 1. Ubicación del Valle de Tumbaco y la estación climatológica La Tola.

\subsection{Climatología}

Las condiciones agroecológicas del valle de Tumbaco se caracterizan por tener dos estaciones climáticas bien diferenciadas: la lluviosa de octubre a mayo, y verano de junio a septiembre; durante los meses de julio y agosto se presentan vientos fuertes, mayor insolación y temperaturas máximas y mínimas; la humedad relativa máxima mensual se registra en el mes de abril con el $79,20 \%$ (mes más lluvioso) y la mínima en el mes de agosto con el $65,70 \%$ (mes más seco); la temperatura máxima varía entre $25,4{ }^{\circ} \mathrm{C}$ y $26,6{ }^{\circ} \mathrm{C}$; la temperatura 
mínima varía entre $4,8{ }^{\circ} \mathrm{C}$ y $7,3{ }^{\circ} \mathrm{C}$; la velocidad del viento máxima se registra en el mes de agosto con 1,70 $\mathrm{m} \mathrm{s}^{-1}$ y la mínima en el mes de abril con $0,50 \mathrm{~m} \mathrm{~s}^{-1}$; el mes con el mayor número de horas de luz es agosto con $7,50 \mathrm{~h} \mathrm{~d}^{-1} \mathrm{y}$ el mes con el menor número de horas de luz es abril con $4,3 \mathrm{~h} \mathrm{~d}^{-1}$ (Tabla 1 ).

Tabla 1. Información climatológica promedio. Período 1986-2018 (INAMHI, 2018)

\begin{tabular}{lccccc}
\hline Mes & $\begin{array}{c}\text { HR } \\
\mathbf{\%}\end{array}$ & $\begin{array}{c}\text { Tmax } \\
{ }^{\mathbf{0}} \mathbf{C}\end{array}$ & $\begin{array}{c}\text { Tmin } \\
{ }^{\mathbf{}} \mathbf{C}\end{array}$ & $\begin{array}{c}\text { Viento } \\
\mathbf{m ~ s}^{-\mathbf{1}}\end{array}$ & $\begin{array}{c}\text { Horas de luz } \\
\mathbf{h ~ d ~ d ~}^{\mathbf{1}}\end{array}$ \\
\hline Enero & 76,7 & 25,7 & 5,9 & 0,8 & 5,8 \\
Febrero & 77,3 & 25,7 & 6,6 & 0,6 & 4,8 \\
Marzo & 78,0 & 25,7 & 7,0 & 0,8 & 4,3 \\
Abril & 79,2 & 25,4 & 7,3 & 0,5 & 4,5 \\
Mayo & 77,3 & 25,5 & 7,0 & 0,7 & 5,1 \\
Junio & 72,1 & 25,6 & 5,8 & 0,9 & 6,0 \\
Julio & 66,7 & 25,6 & 4,8 & 1,5 & 7,1 \\
Agosto & 65,7 & 26,1 & 4,8 & 1,7 & 7,5 \\
Septiembre & 69,3 & 26,6 & 5,0 & 1,1 & 6,2 \\
Octubre & 75,1 & 26,1 & 5,7 & 0,9 & 5,5 \\
Noviembre & 76,8 & 25,8 & 5,8 & 0,6 & 5,3 \\
Diciembre & 75,9 & 25,5 & 6,0 & 0,8 & 5,7 \\
\hline
\end{tabular}

2.3 Métodos para determinar la evapotranspiración de referencia

Penman-Monteith FAO56 (Allen et al., 2006).

$$
E T_{o}=\frac{0,408 \times \Delta \times(R n-G)+\gamma \times \frac{900}{(T+273)} \times U_{2} \times(e s-e a)}{\Delta+\gamma \times\left(1+0,34 \times U_{2}\right)}
$$

Donde: $E T_{\text {o }}$ es la evapotranspiración de referencia $\left(\mathrm{mm} \mathrm{d}^{-1}\right) ; R n$, radiación neta en la superficie del cultivo ( $\mathrm{MJ} \mathrm{m}^{-2} \mathrm{~d}^{-1}$ ); $G$, flujo del calor de suelo $\left(\mathrm{MJ} \mathrm{m}^{-2} \mathrm{~d}^{-1}\right) ; U_{2}$, velocidad del viento $\left(\mathrm{m} \mathrm{s}^{-1}\right)$; ea, presión real de vapor $(\mathrm{kPa})$; es, presión de vapor de saturación; $\Delta$, pendiente de la curva de variación de la presión $\left(\mathrm{kPa}^{\circ} \mathrm{C}^{-1}\right) ; T$, temperatura promedio $\left({ }^{\circ} \mathrm{C}\right) ; \gamma$, constante psicométrica $\left(\mathrm{kPa}^{\circ} \mathrm{C}^{-1}\right) ; Z$, elevación sobre el nivel medio del mar; $\lambda$, calor latente de vaporización $\left(\mathrm{MJ} \mathrm{kg}^{-1}\right)$.

Tanque evaporímetro Clase A (Allen et al., 2006; Doorenbos \& Pruitt, 1977).

$$
\begin{gathered}
E T_{o}=K p \times E o \\
K p=0,5321-3 \times 10^{-4} \times U_{2}+0,0249 \times \operatorname{Ln}(F)+0,0025 \times H R
\end{gathered}
$$

Donde: $K p$ es el coeficiente del tanque evaporímetro; Eo, evaporación del tanque $\left(\mathrm{mm} \mathrm{d}^{-1}\right) ; F$ distancia que existe entre el tanque evaporímetro y el área con cultivo (m). Para la estación climatológica La Tola, el método Snyder presentó los mejores índices inferenciales; el coeficiente promedio fue 0,72 (Ortiz et al., 2018).

Thornthwaite modificado (Thornthwaite, 1948; Camargo et al., 1999).

$$
\begin{gathered}
\text { Tef }=0,35 \times(3 \times T \max -T \mathrm{~min}) \\
I=\sum_{i=1}^{12}\left(\frac{T_{i}}{5}\right)^{1,514} \\
0,49239+0,0179 \times I-0,0000771 \times I^{2}+0,000000675 \times 1 \\
E=1,6 \times\left(\frac{10 \times T e f}{I}\right)^{a} \mathrm{si} \mathrm{T}<26^{\circ} \mathrm{C} \\
E=-415,85+32,24 \times T e f-0,43 \times T e f^{2} \mathrm{si} \mathrm{T}>26^{\circ} \mathrm{C} \\
E T_{o}=E \times \frac{N d \times N h}{360}
\end{gathered}
$$

Donde: $I$ es el índice de calor anual; $T i$, la temperatura promedio mensual $\left({ }^{\circ} \mathrm{C}\right)$; $\mathrm{Tef}$, la temperatura efectiva $\left({ }^{\circ} \mathrm{C}\right) ; E$, la evapotranspiración mensual sin ajustar $\left(\mathrm{mm} \mathrm{m}^{-1}\right) ; N d$, el número de días del mes; $N h$, la duración astronómica del día (h); $E T_{o}$, la evapotranspiración mensual corregida $(\mathrm{mm} \mathrm{mes})^{-1}$.

Hargreaves (Hargreaves \& Samani, 1985).

$E T_{o}=0,0135 \times K T \times R a \times(T+17,8) \times\left(T_{\max }-T_{\min }\right)^{0.5}$ 
Donde: $R a$ es la radiación solar entrante de onda corta $\left(\mathrm{mm} \mathrm{d}^{-1}\right)$; Tmax, la temperatura máxima $\left({ }^{\circ} \mathrm{C}\right) ; \operatorname{Tmin}$, la temperatura mínima $\left({ }^{\circ} \mathrm{C}\right) ; K T, 0,162$ por regiones internas y 0,19 para regiones costeras.

Jensen - Haise (Allen et al., 1990; Landon, 2004).

$$
E T_{o}=R s \times(0,0252 \times T+0,078)
$$

Donde: $R s$ es la radiación solar entrante de onda corta $\left(\mathrm{mm} \mathrm{d}^{-1}\right)$.

Makkink (Allen et al., 1990; Landon, 2004).

$$
E T_{o}=0,61 \times \frac{R s}{\lambda} \times\left(\frac{\Delta}{\Delta+\gamma}\right)-0,12
$$

Priestley and Taylor (Priestley \& Taylor, 1972).

$$
E T_{o}=1,26 \times\left(\frac{\Delta}{\Delta+\gamma}\right) \times(R n-G)
$$

Turc, para HR mayor a 50\%, condición ambiental del Valle de Tumbaco (Tabla 1).

$$
E T_{o}=0,0133 \times\left(\frac{T}{T+15}\right) \times(R s+50)
$$

FAO Radiación (Doorenbos \& Pruitt, 1977).

$b=1,066-0,0013 \times H R+0,045 \times U_{2}-0,0002 \times H R \times U_{2}-0,0000315 \times H R^{2}-0,0011 U_{2}$

$$
E T_{o}=b \times R s \times\left(\frac{\Delta}{\Delta+\gamma}\right)-0,3
$$

Donde: $b$ es el coeficiente de ajuste según la velocidad del viento y la humedad relativa.

Previo a la determinación de $\mathrm{ET}_{\mathrm{o}}$, se realizó un control de calidad de las series de datos para verificar que estas sean independientes, homogéneas y consistentes, con un nivel de significancia del $95 \%$.

Abreviaciones: la $\mathrm{ET}_{0}$ determinada a través del método: Penman-Monteith FAO 56 se define como FA056; tanque evaporímetro como Tan; Thornthwaite modificado como Th; Hargreaves como Ha; Jensen and Haise como JH; Makkink como
Mk; Priestley and Taylor como PT; Turc como Tu y FAO Radiación como Ra.

\subsection{Parámetros inferenciales de evaluación}

Para el análisis entre los diferentes métodos, tomando FAO56 como base, se evaluaron los siguientes índices inferenciales:

Error medio absoluto (MAE, $\mathrm{mm} \mathrm{mes}^{-1}$ ), utilizado para establecer la diferencia que existe entre una variable medida con relación a una estimada, es el promedio de todos los errores absolutos; cuando este valor tiende a cero, el error es menor entre la variable medida y la estimada, el valor óptimo es igual a cero (Chai \& Draxler, 2014).

$$
M A E=\frac{\sum_{i=1}^{n}\left|O_{i}-E_{i}\right|}{n}
$$

Donde: $O$ es el valor observado; $E$, el valor proyectado; $n$, el número de valores en estudio.

Coeficiente de determinación $\left(R^{2}\right)$, calculado a través de regresiones simples, es un índice estadístico utilizado para medir la relación lineal entre dos variables cuantitativas; el valor óptimo debe ser igual a uno y cuando este valor tiende a cero, la correlación entre dos variables es baja; cuando el valor es positivo, la relación es directa y cuando el valor es negativo, la relación es inversa (Samuels, 2014).

$$
R^{2}=\frac{\left[\sum_{i=1}^{n}\left(O_{i}-X\right)\left(E_{i}-E_{m}\right)\right]^{2}}{\left(\sum_{i=1}^{n} O i^{2}-n X^{2}\right) \times\left(\sum_{i=1}^{n} E i^{2}-n E_{m}{ }^{2}\right)}
$$

Donde: $E i$ es el valor proyectado; $E m$, el promedio de los valores proyectados; $X$, el promedio de los valores observados.

Coeficiente de concordancia (D), determina la precisión de los resultados proporcionados por el modelo utilizado para pronosticar un valor observado; cuando este valor tiende a uno, el modelo presenta una mayor precisión (Willmott et al., 2012).

$$
D=1-\frac{\sum_{i=1}^{n}\left(o_{i}-E_{i}\right)^{2}}{\sum_{i=1}^{n}\left(o_{i}-X+E_{i}-X\right)^{2}}
$$

Coeficiente de confiabilidad (C), determina la precisión de los resultados con base en el coeficiente de determinación y el coeficiente de correlación (Silva et al., 2018). 


$$
C=R \times D
$$

Tabla 2. Criterio para interpretar el coeficiente de confiabilidad (Silva et al., 2018)

\begin{tabular}{cl}
\hline Coeficiente de confiabilidad & Clasificación \\
\hline$>0,85$ & Excelente \\
0,76 to 0,85 & Muy bueno \\
0,66 to 0,75 & Bueno \\
0,61 to 0,65 & Intermedio \\
0,51 to 0,60 & Tolerable \\
0,41 to 0,50 & Pobre \\
$<0,40$ & Muy pobre \\
\hline
\end{tabular}

\subsection{Validación del método de mejor ajuste}

El método que presente un mayor índice de confiabilidad se analizará para los años de máxima (2015) y mínima (1988) evapotranspiración anual, para determinar su grado de precisión en condiciones extremas.

\section{Resultados}

$\mathrm{LaET}_{\mathrm{o}}$ determinada por los nueve métodos para las condiciones ambientales del Valle de Tumbaco e información climatológica mensual se presenta en la Tabla 3 y Figura 2a.

Tabla 3. ET $\mathrm{T}_{\mathrm{o}}$ mensual y anual para la estación La Tola con los nueve métodos evaluados

\begin{tabular}{|c|c|c|c|c|c|c|c|c|c|c|c|c|c|}
\hline Métodos & $\mathbf{E}$ & F & M & $\mathbf{A}$ & M & $\mathbf{J}$ & $\mathbf{J}$ & $\mathbf{A}$ & $\mathbf{S}$ & O & $\mathbf{N}$ & D & Total \\
\hline FAO56 & 103,89 & 92,32 & 99,43 & 90,17 & 93,85 & 95,35 & 118,22 & 129,64 & 114,42 & 109,05 & 95,29 & 101,23 & 1242,85 \\
\hline Tan & 92,65 & 82,47 & 80,84 & 75,69 & 84,67 & 101,06 & 123,81 & 136,65 & 111,38 & 92,50 & 81,63 & 88,89 & 1152,25 \\
\hline Th & 132,47 & 121,72 & 128,75 & 121,40 & 127,33 & 126,73 & 134,74 & 138,79 & 137,83 & 136,44 & 129,38 & 130,37 & 1565,97 \\
\hline $\mathrm{Ha}$ & 149,80 & 143,48 & 154,35 & 142,59 & 140,09 & 132,53 & 140,98 & 151,34 & 155,33 & 157,15 & 146,03 & 146,11 & 1759,78 \\
\hline $\mathrm{JH}$ & 107,61 & 97,13 & 101,04 & 96,29 & 98,44 & 96,51 & 107,86 & 118,21 & 110,16 & 107,98 & 99,65 & 104,15 & 1245,03 \\
\hline PT & 112,17 & 103,27 & 108,83 & 103,00 & 101,97 & 97,56 & 107,10 & 117,10 & 112,72 & 113,79 & 105,41 & 108,64 & 1291,54 \\
\hline $\mathrm{Mk}$ & 95,07 & 84,81 & 87,64 & 83,54 & 85,67 & 85,62 & 97,41 & 105,97 & 97,48 & 95,16 & 88,17 & 92,23 & 1098,76 \\
\hline $\mathrm{Tu}$ & 106,69 & 100,75 & 108,25 & 104,74 & 107,99 & 102,79 & 104,66 & 105,44 & 103,22 & 106,92 & 103,12 & 106,40 & 1260,97 \\
\hline $\mathrm{Ra}$ & 117,34 & 102,99 & 106,03 & 98,99 & 103,88 & 109,41 & 133,33 & 147,57 & 129,58 & 119,40 & 107,53 & 114,29 & 1390,35 \\
\hline
\end{tabular}

Considerando el mes de máxima ET (agosto: Tabla 3, columna 9) y el método FAO56 como base, el tanque evaporímetro proporcionó un valor de $\mathrm{ET}_{\text {o }}$ superior del 5,41\%; Thornthwaite modificado un valor superior del $7,06 \%$; Hargreaves un valor superior del 16,74\%; Jensen and Haise un valor inferior del 9,67\%; Priestley and Taylor un valor inferior del 9,67\%; Makkink un valor inferior del 18,26\%; Turc un valor inferior del $18,67 \%$ y FAO Radiación un valor superior del 13,83\%.

Considerando el total anual de $\mathrm{ET}_{\mathrm{o}}$ (Tabla 3, columna 14) y el método FAO56 como base, el tanque evaporímetro proporcionó un valor de $\mathrm{ET}_{\mathrm{o}}$ inferior del 7,29\%; Thornthwaite modificado un valor superior del 26,0\%; Hargreaves un valor superior del $41,59 \%$; Jensen and Haise un valor mayor del $0,18 \%$; Priestley and Taylor un valor superior del 3,92\%; Makkink un valor inferior del 11,59\%; Turc un valor superior del 1,46\% y FAO Radiación un valor superior del $11,87 \%$.

El método del tanque evaporímetro para información climatológica de toda la serie histórica de datos presentó un desempeño pobre (Tabla 4); para el año de máxima ET índices intermedios (Tabla 5) y para el año de mínima $\mathrm{ET}_{\mathrm{o}}$ un índice de confiabilidad muy pobre (Tabla 5). En otras zonas de la región, como Sao Paulo (Brasil), este método presentó mejores resultados que FAO56 considerando ET determinada a través de lisímetros (Sentelhas \& Folegatti, 2003).

El método Thornthwaite modificado presentó un índice de confiabilidad muy pobre tanto para toda la serie de datos como para el año de máxima y mínima $\mathrm{ET}_{\mathrm{o}}$ (Tablas 4 y 5). Este método es muy utilizado en el Ecuador en estudios de balances hídricos (CISPDR, 2016). 
Tabla 4. Parámetros inferenciales. Serie total de datos

\begin{tabular}{lccccl}
\hline Métodos & MAE & $\mathbf{R}^{2}$ & $\mathbf{D}$ & $\mathbf{C}$ & Clasificación \\
\hline Tan & 18,86 & 0,64 & 0,66 & 0,42 & Pobre \\
Th & 28,93 & 0,44 & 0,45 & 0,20 & Muy pobre \\
Ha & 27,71 & 0,26 & 0,26 & 0,07 & Muy pobre \\
JH & 11,00 & 0,73 & 0,74 & 0,54 & Tolerable \\
PT & 13,15 & 0,66 & 0,64 & 0,42 & Pobre \\
Mk & 13,88 & 0,81 & 0,72 & 0,58 & Tolerable \\
Tu & 14,81 & 0,06 & 0,34 & 0,02 & Muy pobre \\
Ra & 12,86 & 0,88 & 0,78 & 0,69 & Bueno \\
\hline
\end{tabular}

El método Hargreaves presentó un índice de confiabilidad muy pobre tanto para toda la serie de datos como para el año de máxima y mínima $\mathrm{ET}_{\text {o }}$ (Tablas 4 y 5); en otras zonas de la región presentó índices inferenciales de alta precisión como en Campos Dos Goytacazes en Rio de Janeiro en Brasil (Lázaro et al., 2012), en la región del libertador general Bernardo O'Higgins en Chile (Sánchez \& Carvacho, 2011) y en la cuenca andino-amazónica peruana (Lavado Casimiro et al., 2015).

El método Jensen and Haise para información climatológica de toda la serie histórica de datos presentó un desempeño tolerable (Tabla 4), mientras que para el año de máxima y mínima $\mathrm{ET}_{\mathrm{o}}$ índices muy pobres (Tabla 5).

El método Priestley and Taylor para información climatológica de toda la serie histórica de datos presentó un desempeño pobre (Tabla 4), para el año de máxima ETo un índice muy pobre y para el año de mínima $\mathrm{ET}_{\mathrm{o}}$, índices pobres (Tabla 5); en la provin- cia del Azuay (Ecuador) presentó índices confiables para estimar ET en la microcuenca del río Quinuas (Contreras, 2015), así como, índices de alta precisión en Campos Dos Goytacazes en Rio de Janeiro en Brasil (Lázaro et al., 2012).

El método Makkink para información climatológica de toda la serie histórica de datos presentó un desempeño tolerable (tabla 4); para el año de máxima $\mathrm{ET}_{\mathrm{o}}$ un índice muy pobre (Tabla 5), y para el año de mínima $\mathrm{ET}_{\mathrm{o}}$ un índice de confiabilidad pobre (Tabla 5); este método presentó índices de alta precisión en Campos Dos Goytacazes en Rio de Janeiro (Lázaro et al., 2012).

El método Turc presentó un índice de confiabilidad muy pobre tanto para toda la serie de datos como para el año de máxima y mínima $\mathrm{ET}_{\mathrm{o}}$, por el contrario, en la región del libertador general Bernardo O'Higgins (Chile) presentó índices de alta precisión (Sánchez \& Carvacho, 2011).

El método FAO Radiación presentó un coeficiente de confiabilidad bueno para toda la serie de datos (Tabla 4), excelente para el año de máxima ET。 (Tabla 5), y para el año de mínima $\mathrm{ET}_{\mathrm{o}}$ un coeficiente muy pobre (Tabla 5).

Para el año de ET máxima (2015), FAO56 proporcionó un total anual de $1.550,78 \mathrm{~mm}$ y FAO Radiación 1.571,69 mm, con una diferencia de 20,91 mm (1,35\%); para el año de $\mathrm{ET}_{\text {o }}$ mínima (1988), FAO56 proporcionó $1.049,47 \mathrm{~mm}$ y FAO Radiación $1.278,72 \mathrm{~mm}$, con un exceso de 229,25 $\mathrm{mm}(21,84 \%)$.

Tabla 5. Validación del método FAO Radiación para el año de máxima y mínima ET。

\begin{tabular}{|c|c|c|c|c|c|c|c|c|c|c|}
\hline \multirow{2}{*}{ Métodos } & \multicolumn{5}{|c|}{ Año 2015} & \multicolumn{5}{|c|}{ Año 1988} \\
\hline & MAE & $\mathbf{R}^{2}$ & D & C & Clasificación & MAE & $\mathbf{R}^{2}$ & D & C & Clasificación \\
\hline Tan & 30,06 & 0,91 & 0,68 & 0,62 & Intermedio & 15,90 & 0,19 & 0,53 & 0,10 & Muy pobre \\
\hline Th & 17,41 & 0,37 & 0,60 & 0,22 & Muy pobre & 42,18 & 0,20 & 0,28 & 0,06 & Muy pobre \\
\hline $\mathrm{Ha}$ & 20,18 & 0,08 & 0,40 & 0,03 & Muy pobre & 40,57 & 0,38 & 0,31 & 0,12 & Muy pobre \\
\hline JH & 19,36 & 0,56 & 0,58 & 0,32 & Muy pobre & 14,85 & 0,50 & 0,61 & 0,31 & Muy pobre \\
\hline PT & 20,27 & 0,27 & 0,51 & 0,14 & Muy pobre & 17,34 & 0,82 & 0,58 & 0,48 & Pobre \\
\hline $\mathrm{Mk}$ & 34,20 & 0,64 & 0,51 & 0,33 & Muy pobre & 4,81 & 0,54 & 0,80 & 0,43 & Pobre \\
\hline $\mathrm{Tu}$ & 23,74 & 0,07 & 0,43 & 0,03 & Muy pobre & 21,44 & 0,10 & 0,40 & 0,04 & Muy pobre \\
\hline $\mathrm{Ra}$ & 6,51 & 0,90 & 0,97 & 0,87 & Excelente & 19,10 & 0,60 & 0,59 & 0,35 & Muy pobre \\
\hline
\end{tabular}



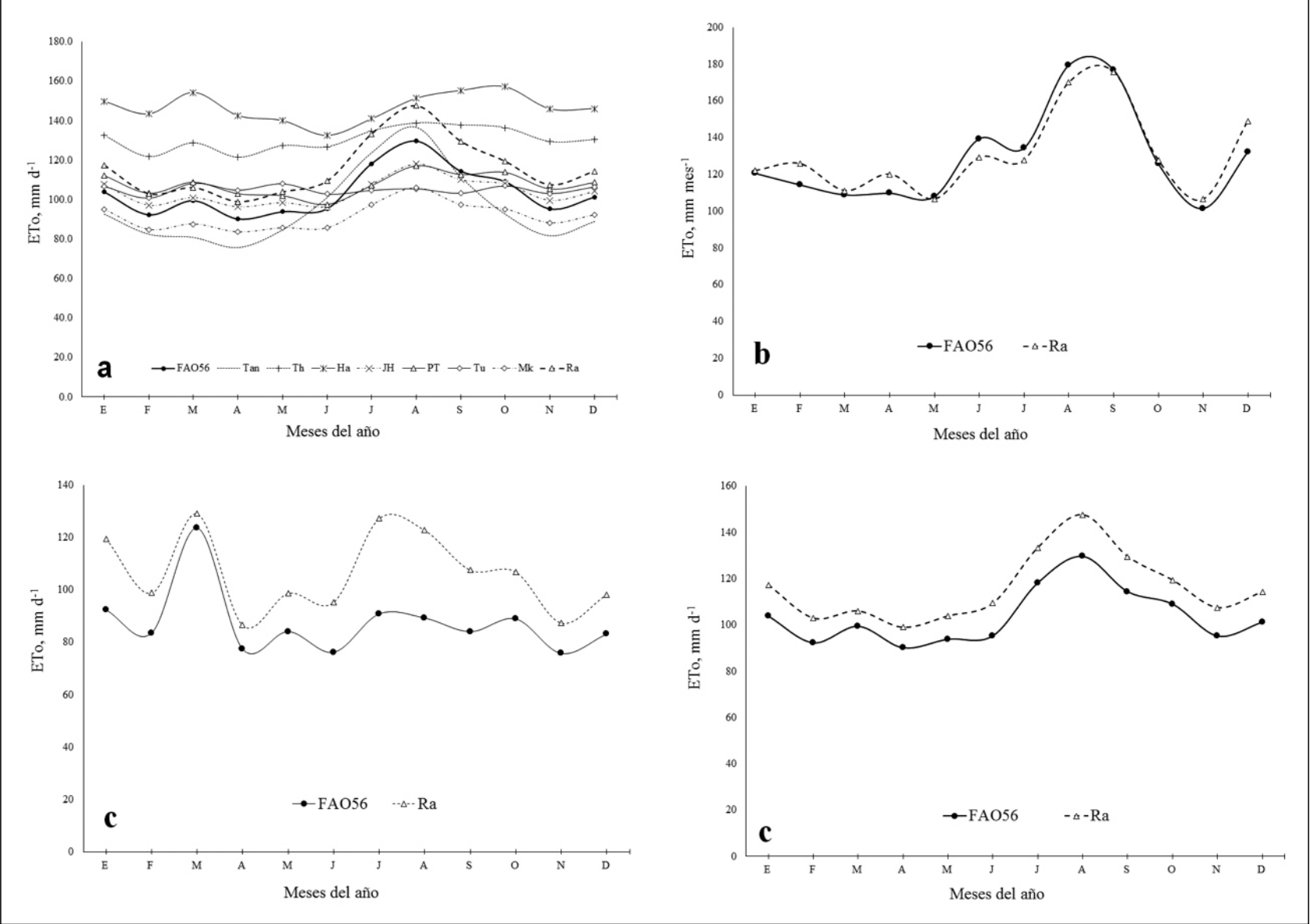

Figura 2. Evapotranspiración de referencia: (a) promedio mensual multianual de los nueve métodos, (b) ET óxíxima anual (2015), (c) ET mínima anual (1988), y (d) ET $T_{\mathrm{o}}$ promedio multianual (serie de 32 años).

El método FAO Radiación para el año con mayor $\mathrm{ET}_{\mathrm{o}}$ (2015), subestima $\mathrm{ET}_{\mathrm{o}}$ en los meses de junio, julio y agosto, y sobreestima en febrero, abril y diciembre (Figura 2b), siendo diciembre el mes con máxima variación (12,66\%); para el año con ET mínima (1988), sobreestima $\mathrm{ET}_{\mathrm{o}}$ durante todo el año (Figura 2c), siendo julio el mes en el que presenta la máxima variación (40,16\%); para valores de $\mathrm{ET}_{\text {。 }}$ promedio de la serie histórica, sobreestima ETo durante todo el año, siendo junio en donde se presenta la máxima variación con un 14,75\% (Figura 2d).

\section{Discusión}

Los métodos para calcular $\mathrm{ET}_{\mathrm{o}}$ como: FAO56, tanque evaporímetro, Thornthwaite, Hargreaves, Jensen and Haise y Turc presentan diferencias sustanciales en los procedimientos matemáticos para determinar ET. Los métodos FAO56, FAO Radiación, Makkink, Priestley and Taylor, se derivan de la ecuación original de Penman.

El método FAO56, a diferencia de los otros métodos, es un modelo aerodinámico completo, el cual integra en su fórmula de cálculo todas las variables que intervienen en el proceso de $\mathrm{ET}_{\mathrm{o}}$ tal como la resistencia aerodinámica, la resistencia superficial del cultivo y el albedo (Allen et al., 2006), presentando una alta correlación cuando ha sido contrastado con lisímetros, tanto en zonas áridas como húmedas y templadas (Allen et al., 1990).

El método del tanque evaporímetro presentó índices inferenciales que varían de muy pobre a intermedio; a pesar de su sencillez, requiere de una operación y mantenimiento rigurosos para obtener lecturas confiables de evaporación, considerando que el color del tanque, lámina de agua, dificultad para leer la evaporación durante las lluvias, entre otros factores, influyen directamente en el balance energético, y por lo tanto en los valores registrados de evaporación.

El método Thornthwaite modificado presentó índices inferenciales muy pobres, debido a que los coeficientes están calibrados para condiciones ambientales de Estados Unidos, en donde existen cuatro estaciones climáticas bien diferenciadas (otoño, 
invierno, primavera y verano), presentándose temperaturas bajo cero en invierno (enero), situación muy diferente a la que ocurre en el valle de Tumbaco, en donde se registran temperaturas máximas de $25,84^{\circ} \mathrm{C}$ y mínimas de $5,95^{\circ} \mathrm{C}$ en el mes de enero (Tabla 1 ).

El método Hargreaves presentó índices inferenciales muy pobres, debido a que este método se desarrolló para condiciones ambientales de Estados Unidos, en donde se presentan días con 4 horas de luz en invierno y de hasta 16 horas en verano, mientras que en el Ecuador existen 12 horas de luz durante los 365 días del año.

El método Jensen and Haise presentó índices inferenciales que varían de muy pobre a tolerable. El método Makkink presentó mejores índices inferenciales que el método Priestley and Taylor, debido a que los valores del coeficiente "a" son diferentes (ecuaciones 10 y 11), para Makkink este valor es de 0,61 y para Priestley and Taylor de 1,26. Por otro lado, mientras que en el método Makkink se sustrae una constante de $0,12 \mathrm{~mm} \mathrm{~d}^{-1}$ de ET, el método Priestley and Taylor utiliza la radiación neta y el flujo del calor del suelo para determinar $\mathrm{ET}_{\mathrm{o}}$.

El método Turc presentó índices inferenciales muy pobres, debido a que los coeficientes se ajustaron para la climatología del oeste de Europa (Landon, 2004), muy diferente a las condiciones climatológicas ecuatoriales, en donde existen dos estaciones climáticas bien diferenciadas (invierno y verano); en invierno, las lluvias se presentan por el efecto atmosférico que produce la corriente cálida de El Niño y, en verano, el clima se encuentra afectado por la corriente fría de Humboldt. Por otro lado, las cordilleras oriental y occidental de los Andes que rodean al valle de Tumbaco también influyen en el clima, con la presencia de los nevados (Cotopaxi, Antisana, entre otros), los cuales producen temperaturas muy bajas o heladas en verano. Asimismo, en los meses de julio y agosto, la

\section{Referencias}

Allen, R., Burman, R., \& Jensen, M. (1990). Evapotranspiration and Irrigation Water Requirements. New York: American Society of Civil Engineers.

Allen, R., Pereira, L., Raes, D., \& Smith, M. (2006). Evapotranspiración del cultivo. Roma: FAO. presencia de los vientos incrementa la tasa de evapotranspiración, llegando a ser ésta incluso mayor a la $\mathrm{ET}_{\mathrm{o}}$ que se registra en la Costa ecuatoriana.

A pesar de que los métodos FAO56 y FAO Radiación se basan en el método original de Penman para estimar $\mathrm{ET}_{\mathrm{o}}$, FAO Radiación presentó índices de confiabilidad que varían de muy pobre a excelente, variación asociada con las variables utilizadas en el cálculo, así como, en el ajuste del coeficiente b que utiliza FAO Radiación para estimar la evapotranspiración de referencia (ecuación 15). FAO Radiación no utiliza las siguientes variables para estimar $\mathrm{ET}_{0}$ : radiación neta en la superficie del cultivo y flujo de calor del suelo. Por otro lado, el coeficiente b está calibrado para condiciones ambientales de Estados Unidos.

\section{Conclusiones}

Con base en los índices inferenciales MAE, $\mathrm{R}^{2}$, D y C, el método FAO Radiación presentó una precisión buena; Jensen and Haise, Makkink y Priestley and Taylor, proporcionaron una precisión tolerable; el tanque evaporímetro, Hargreaves, Turc y Thornthwaite, índices inferenciales muy pobres.

A pesar de que los métodos Jensen and Haise y Turc proporcionan valores anuales de $\mathrm{ET}_{0}$ cercanos a FAO56, las diferencias sustanciales en ET mensual, hacen que estos métodos no presenten índices inferenciales de mayor precisión.

Los métodos basados en la temperatura y radiación solar, subestiman ET durante los meses de julio y agosto, debido a que no incorporan en su fórmula la velocidad del viento, cuyos valores oscilan entre 1,1 y $1,7 \mathrm{~m} \mathrm{~s}^{-1}$, afectando directamente a la tasa de evapotranspiración.

Para estimar $\mathrm{ET}_{\mathrm{o}}$ con las condiciones ambientales del Valle de Tumbaco debe utilizarse el método FAO56.

Bhabagrahi, S., Imtisenla, W., Bidyut, D., \& Bhagwati, B. (2012). Standardization of Reference Evapotranspiration Models for a Subhumid Valley Rangeland in the Eastern Himalayas. Journal of Irrigation and Drainage Engineering, 138, 880-895. 
Camargo, A., Marín, F., Sentelhas, P., \& Picini, A. (1999). Adjust of the Thornthwaite's method to estimate the potential evapotranspiration for arid and superhumid climates, based on daily temperature amplitude. Revista Brasileira de Agrometeorología, 7(2), 251-257.

Chai, T., \& Draxler, R. R. (2014). Root mean square error (RMSE) or mean absolute error (MAE)? Arguments against avoiding RMSE in the literature. Geoscientific Model Development, 7, 12471250. https://doi.org/10.5194/gmd-7-1247-2014

CISPDR - Changjiang Institute of Survey, Planning, Desing and Research. (2016). Plan nacional de la gestión integrada e integral de los recursos hídricos de las cuencas y micro cuencas hidrográficas del Ecuador. Ecuador: Secretaría Nacional del Agua.

Contreras, J. (2015). Análisis comparativo de cuatro modelos de evapotranspiración de referencia en la microcuenca del río Quinuas. Cuenca: Universidad de Cuenca.

Doorenbos, J., \& Pruitt, W. (1977). Guidelines for predicting crop water requirements. Roma: FAO.

Hargreaves, G., \& Samani, Z. (1985). Reference crop evapotranspiration from temperature. Applied Engineering in Agriculture, 1(2), 96-99. https:// doi.org/10.13031/2013.26773

INAMHI. (2018). Información climatológica proporcionada por el INAMHI. Quito, Ecuador.

Landon, R. (2004). An evaluation of reference evapotranspiration models in Louisiana. United States: Louisiana State University.

Lavado Casimiro, W.S., Lhomme, J.-P., Labat, D., Guyot, J.-L., \& Boulet, G. (2015). Estimación de la evapotranspiración de referencia (FAO-56 Penman - Monteith) con limitados datos climáticos en la cuenca andina amazónica peruana. Revista Peruana Geo-Atmosférica, 4, 31-43.

Lázaro, F., Célia, M., \& Otto, C. (2012). Evaluation of six empirical evapotranspiration equations - Case study: campos dos Goytacazas. Revista Brasileira de Meteorología, 27(3), 272-280.https://doi.org/10.1590/S010277862012000300002
Ortiz, R. Tamayo, Ch. Chile, M. \& Méndez, A. (2018). Coeficiente del tanque evaporímetro Clase A para estimar la evapotranspiración de referencia para el valle de Tumbaco. Siembra, 5(1), 16-25.

Priestley, C., \& Taylor, R. (1972). On the assessment of surface heat flux and evaporation using large-scale parameters. Atmospheric Physics, 100(2), 81-92.

Samuels, P. (2014). Pearson Correlation. Disponible en: http://www.statstutor.ac.uk/resources/ uploaded/pearsoncorrelation3.pdf

Sánchez, M., \& Carvacho, L. (2011). Comparación de ecuaciones empíricas para el cálculo de la evapotranspiración de referencia en la región del libertador general Bernardo O'Higgins, Chile. Revista de Geografía Norte Grande, 50, 171-186. http://dx.doi.org/10.4067/S071834022011000300010

Sentelhas, P., \& Folegatti, M. (2003). Class A pan coefficients $(\mathrm{Kp})$ to estimate daily reference evapotranspiration (ET). Revista Brasileira de Engenharia Agricola e Ambiental, 7(1), 111-115. https://doi.org/10.1590/S141543662003000100018

Silva, G. H. da, Dias, S. H. B., Ferreira, L. B., Santos, J. É. O., \& Cunha, F. F. da. (2018). Performance of different methods for reference evapotranspiration estimation in Jaíba, Brazil. Revista Brasileira de Engenharia Agrícola e Ambiental, 22(2), 83-89. https://doi.org/10.1590/1807-1929/agriambi. v22n2p $83-89$

Thornthwaite, C. (1948). An approach toward a Rational Classification of Climate. American Geographical Society, 38(1), 55-94.

Willmott, C., Robeson, S., \& Matsuura, K. (2012). A refined index of model performance. International Journal of Climatology, 32, 2088-2094. https://doi.org/10.1002/joc.2419 